\title{
Physical functionalization of multi-walled carbon nanotubes for enhanced dispersibility in aqueous medium
}

\author{
Mohamed Youssry ${ }^{1}$ (D) Maisa Al-Ruwaidhi ${ }^{1} \cdot$ Mahdiyeh Zakeri $^{1} \cdot$ Mohadese Zakeri $^{1}$
}

Received: 11 May 2019 / Accepted: 12 February 2020 / Published online: 7 March 2020

(C) The Author(s) 2020

\begin{abstract}
Noncovalent functionalization of multi-walled carbon nanotubes (MWCNTs) by anionic surfactants (sodium dodecylbenzene sulfonate; SDBS and sodium cholate; SC) results in stable aqueous dispersions. SDBS showed better dispersing efficiency at lower concentration than that of SC due to the stabilizing $\pi-\pi$ stacking induced by the presence of benzene ring in the tail of SBDS. The hemimicelle formation on the surface of nanotubes is likely to be the dominant dispersion mechanism that imparts higher colloidal stability for the MWCNTs in aqueous medium.
\end{abstract}

Keywords Multi-walled carbon nanotubes $\cdot$ Aqueous dispersions $\cdot$ Physical functionalization $\cdot$ Colloidal stability

\section{Introduction}

Carbon nanotubes (CNTs) are unique kind of carbonaceous nanomaterials which are characterized by excellent electrical and mechanical properties making them intriguing component in composite reinforcement materials [1], chemical sensors [2], supercapacitors [3], and other energy storage systems [4, 5]. The CNT is viewed as concentric cylinders of graphene sheets rolled up in a hollow rodlike morphology with high aspect ratio $(d / L ; d$ and $L$ denote the diameter and length of nanotube, respectively). Depending on the number of rolled sheets, CNT can be commonly differentiated to three types: (i) single-walled carbon nanotube (SWCNT) which is formed from a single-rolled sheet of graphene with diameter of the order of $1 \mathrm{~nm}$ and length of up to $\mathrm{cm} \mathrm{[6],} \mathrm{(ii)} \mathrm{double-walled}$ carbon nanotube (DWCNT) composed of two rolled sheets giving an average diameter of $2 \mathrm{~nm}$ and length scale up to $10 \mu \mathrm{m}$ [7], and (iii) multi-walled carbon nanotube (MWCNT) which consists of an array of graphene sheets with typical diameter between 2 and $100 \mathrm{~nm}$ and a length of tens of $\mu \mathrm{m}$ [8].

Generally, CNTs have a crystalline structure formed by hexagonal network of benzene rings with carbons having $\mathrm{sp}^{2}$ hybridization [9]. However, the number of defects, due

Mohamed Youssry

myoussry@qu.edu.qa

1 Department of Chemistry and Earth Sciences, College of Arts and Sciences, Qatar University, 2713 Doha, Qatar to polygonal structures, at the endcaps and sidewall of the nanotubes causes them to curve forming seamless structure with $\mathrm{sp}^{3}$ defects lines in the $\mathrm{sp}^{2}$ network which, of course, affect the properties (e.g., electrical properties) of the CNTs [10]. Therefore, CNTs have a unique graphitic structure with mixture of metallic and semiconducting characters [11] depending on the diameter and chirality of nanotubes. For instance, MWNTs showed a high degree of graphitization with an intrinsic electrical conductivity of about $1.85 \mathrm{kS} \mathrm{cm}^{-1}$ along the long axis [12].

Essentially, the CNTs are strongly hydrophobic materials with some degree of hydrophilicity originating from oxygenbased functional groups (e.g., carboxylic, hydroxyl, phenolic, lactone, etc.) randomly distributed on the surface of nanotubes [13]. The concentration of surface oxygen groups varies according to the synthesis methodology or manufacturer, but it is not enough to impose electrostatic stabilization for the suspension of CNTs in aqueous media. Instead, the van der Waals attraction forces dominate leading to aggregation (bundling) of nanotubes and formation of larger agglomerates so that sedimentation occurs [14]. In addition, the electric current only flows on the outermost tubes in a bundled CNTs, while the inner tubes do not contribute significantly to the current [15] resulting in potential loss of electronic wiring. Unfortunately, this behavior renders CNT dispersion a challenging risk and restrains their potential applications in many fields especially the formulation of uniform or homogenous electrodes for electrochemical energy storage systems (EESS) such as ion batteries and redox flow cells [16, 17]. 
Back to 5000 years, the ancient Egyptian used sugar solution to "unbundle" the agglomerated carbon blacks; thereby, they could overcome the van der Waals forces and obtain homogenous stable inks. Recently, many approaches, including mechanical treatment and physical (noncovalent) and chemical (covalent) functionalization, have been developed to overcome the attractive forces and then enhance the dispersion stability of various types of carbon nanotubes.

Mechanical methods such as ultrasonication, high-shear mixing, or combined methods have been commonly used to disperse CNTs in polar solvents [18-20]. However, upon removal of external stress, the CNTs reconfigure themselves to re-agglomerate, and coagulation/sedimentation occurs [21]. In addition, prolonged exposure to mechanical stress seriously shortens the nanotubes as well as induces more structural defects on the sidewall of nanotubes [18, 22-24] which significantly might alter the electrical conductivity of suspensions.

Physical adsorption of amphiphilic molecules (such as surfactants and some kinds of polymers) with the aid of mild sonication resulted in homogenous stable CNT suspensions stabilized by either electrostatic (for ionic surfactants) or steric (for nonionic surfactant of polymers) repulsion forces. Wrapping of nonionic surfactants [7, 25, 26] or polymer molecules [27-30] on the CNTs enhanced the CNT dispersibility but significantly minimize the electrical conductivity of suspensions. Alternatively, anionic surfactants such as sodium dodecyl sulfate (SDS) [25, 31-36], sodium dodecyl benzene sulfonate (SDBS) [25, 35, 37-44], and sodium deoxycholate (SDC) [42, 43] have been extensively used as dispersing agents in aqueous suspensions of CNTs. The quality of dispersion is dependent on the surfactant structure and relative concentration of CNTs and the surfactant. For instance, starlike surfactants presented good dispersibility for MWCNTs in aqueous media [45]. There exists a discrepancy in the literature for the optimum surfactant concentration for improved dispersibility $[31,33,46]$. However, there is a kind of qualitative agreement that the surfactant concentration should be 10-100 times above its cmc [47] and that the surfactant concentration nearly equals the CNT concentration [26] for production of homogenous stable CNT suspensions. Therefore, the optimum dispersion is obtained at intermediate concentration of surfactant $[31,33]$ where the nanotubes are unbundled as a consequence of adsorption of surfactants' hydrophobic tails on the nanotubes surface leaving the hydrophilic headgroups extended far into the aqueous medium producing long-ranged electrostatic repulsion forces [48, 49]. When the surfactant concentration increases, the formation of micelles $[26,33,46]$ or monolayers [49] additionally stabilize the suspension through steric stabilization. (High surfactant concentration resulted in depletion-induced aggregation of nanotubes [25]).

The surfactant structure plays an important role in MWCNT dispersibility. It has been found that the $\pi$-like stacking of benzene rings in SDBS benefits the binding of surfactant with CNTs [37, 40, 44]. In comparison, SDC showed preferential adsorption to the nanotubes over SDBS due to stronger hydrophobic interactions with the linked cholesteric moiety in SDBS compared to the single alkyl chain of SDBS [43]. In addition, the absence of hydroxyl group renders SDC as a better dispersing agent than sodium cholate [42, $50]$.

\section{Experimental}

\subsection{Materials}

Multi-walled carbon nanotubes (MWCNTs; purity > $90 \%)$, sodium dodecylbenzene sulfonate (SDBS; $\mathrm{C}_{18} \mathrm{H}_{29} \mathrm{NaO}_{3} \mathrm{~S}$ ), and hydrated sodium cholate ( $\mathrm{SC} ;>$ $\left.99 \% ; \mathrm{C}_{24} \mathrm{H}_{39} \mathrm{NaO}_{5} \cdot \mathrm{xH}_{2} \mathrm{O}\right)$ were purchased from SigmaAldrich, USA. The diameter $(d)$ and length $(L)$ of nanotubes were $110-170 \mathrm{~nm}$ and 5-9 $\mu \mathrm{m}$, respectively, as provided by Sigma-Aldrich, USA. The materials were used without further purifications, and the number of water molecules $(x)$ in the sodium cholate was estimated from the thermogravimetric analysis (TGA from PerkinElmer; data not shown). The weight loss percentage of $15.94 \%$ corresponds to 4.53 molecules of water per molecule of sodium cholate. Therefore, the molar mass of hydrated sodium cholate is $512.1 \mathrm{~g} / \mathrm{mol}$.

\subsection{Preparation of dispersions}

Stock solutions of two anionic surfactants, sodium dodecylbenzene sulfonate (SDBS) and sodium cholate (SC), were prepared by dissolving appropriate masses of surfactants in distilled water at the room temperature (ca. 22-23 ${ }^{\circ} \mathrm{C}$ ). This temperature is above the Krafft temperature of both surfactants [51] so that the stock surfactant solutions remain stable at room temperature. Three stock MWCNT dispersions were prepared by dispersing constant amount of MWCNT at $0.80 \mathrm{mg}$ in $5 \mathrm{~mL}$ surfactant solutions (at 5, 10, and $30 \mathrm{mM}$ SDBS or SC) using bath sonicator (Barason 2800) for $8 \mathrm{~h}$. The temperature is kept constant below $30^{\circ} \mathrm{C}$ by adding ice to the water bath of sonicator.

For UV-Vis and zeta potential measurements, the stock dispersions were diluted by distilled water and/or diluted surfactant solutions keeping the MWCNT concentration at $0.133 \mathrm{mg}$ for $3 \mathrm{~mL}$ of solutions and varying the surfactant concentration from 0.717 to $150 \mathrm{mM}$. Again, the diluted dispersions were homogenized by sonication for $30 \mathrm{~min}$ and then left to equilibrate for $15 \mathrm{~min}$ before measurements. 
Fig. 1 Transmission electron micrographs of multi-walled carbon nanotubes
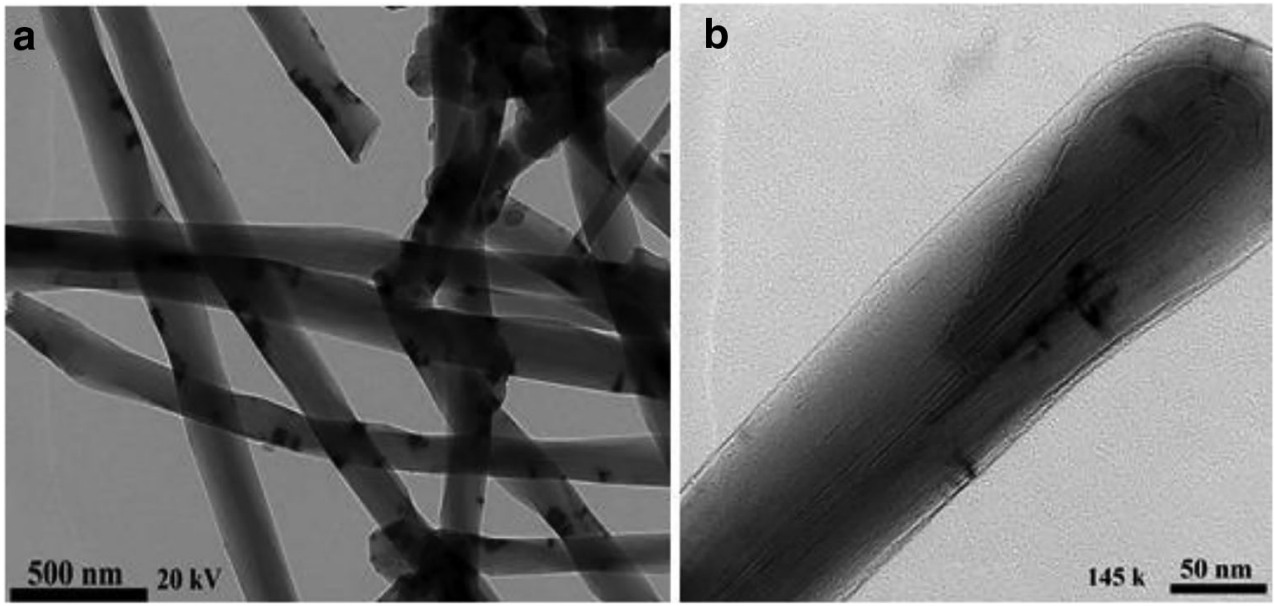

\subsection{UV-Visible spectroscopy}

The UV-Vis measurements were conducted in Agilent Cary 8453 spectrophotometer (with photodiode array) in the frequency range of 230-1100 nm, using 1-cm quartz cuvette. The absorption spectra were collected at room temperature $\left(22-23{ }^{\circ} \mathrm{C}\right)$. Beer-Lambert law relates the absorbance $(A)$ to the concentration $(C)$ through Beer's law: $A=\varepsilon b C$, where $b$ is the path length $(=1 \mathrm{~cm})$ and $\varepsilon$ is the molar absorptivity. Rationally, the absorbance increases as the concentration of nanotubes increases showing a linear relationship. However, Beer's equation correctly holds over defined concentration range beyond which the Beer-Lambert law deviates from linearity at strong absorbance [52], i.e., high MWCNT concentration. Therefore, highly diluted dispersions were prepared from the stock dispersions to keep the measured absorbance $A$ in the range of 0.1 to 2 in the entire wavelength range. In order to eliminate the interference between the absorption of surfactant and MWCNT, the subtraction method $[33,53]$ was followed where an aqueous surfactant solution with the same surfactant concentration was used as reference (blank) before recording the spectrum of its MWCNT dispersion.

\subsection{Zeta potential}

The quality of the nanotube dispersions was estimated by measuring the zeta potential of dispersions by Zetasizer Nano ZSP, Malvern, using light scattering method. Adsorption of charged molecules on MWCNT surfaces affects the zeta potential, because an effective charged molecule appears on the coated tube; the surfactant-coated nanotubes are surrounded by a layer of adsorbed surfactant molecular ions, which is surrounded by a mobile counterions with more diffuse zone; therefore, the electrical double layer formed and the zeta potential can be measured.

Carbon nanotubes commonly has negatively charged surfaces due to some functional oxide surface groups such as carboxylic, hydroxyl groups, etc.; therefore they showed negative zeta potentials. The measuring of zeta potential is an indicator of stability; if the absolute value zeta potential is greater than $15 \mathrm{mV}$, then the colloidal particles are stable due to electrostatic forces. This means that a stable nanotube surfactant dispersion should have a large zeta potential, which indicate that an increase of adsorbed surfactants per unit area of MWCNT surfaces is required.
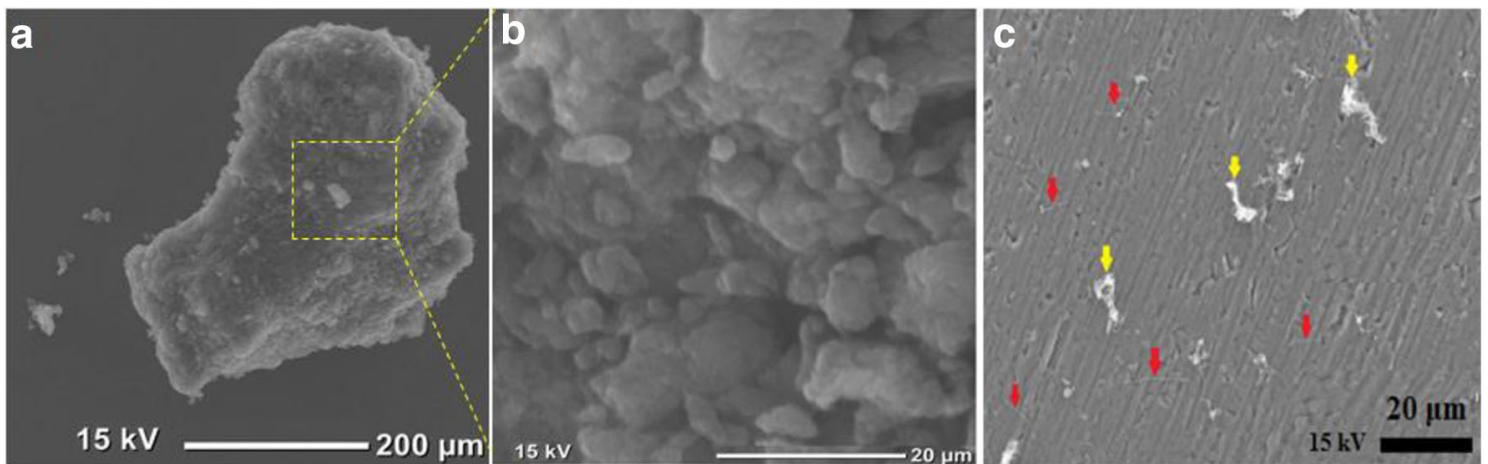

Fig. 2 Scanning electron micrographs of carbon nanotubes in their dry powder $(\mathbf{a}, \mathbf{b})$ and dispersion in sodium cholate solution (c): the red and yellow arrows correspond, respectively, to dispersed tubes and small bundles

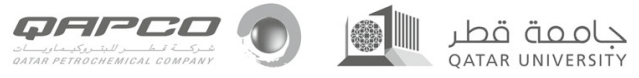




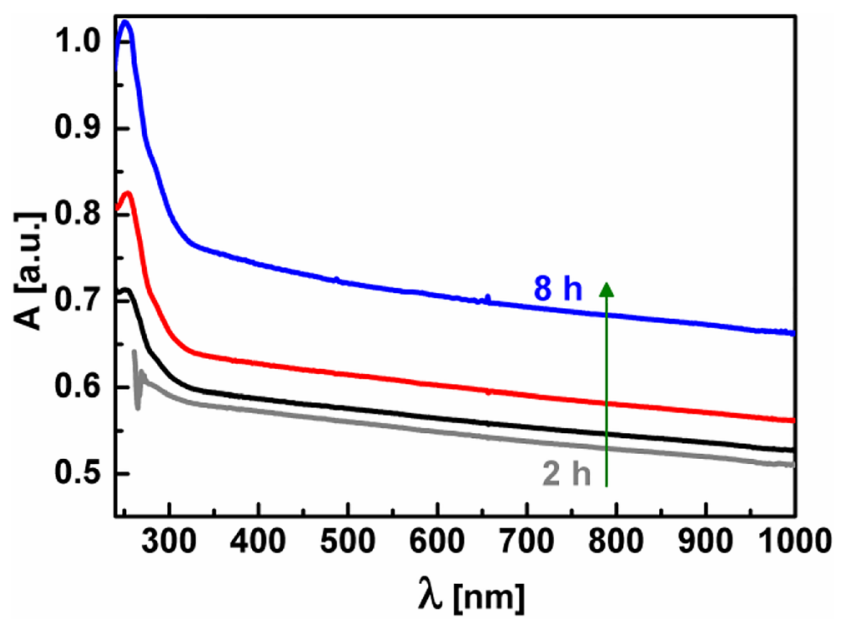

Fig. 3 Absorption spectra (absorbance A versus wavelength $\lambda$ ) of SDBSMWCNT dispersion at different sonication times (from 2 to $8 \mathrm{~h}$ )

\subsection{Electron microscopy}

Transmission electron microscope (TEM; Tecnica) was employed to image the microstructure and morphology of carbon nanotubes. Traces of MWCNTs were dispersed in ethanol and then placed on carbon grids and dried before loading in the instrument. Micrographs were recorded on a Gatan CCD camera and analyzed by Digital Micrograph software. Scanning electron microscope (SEM; JOEL JCM 6000) was used to investigate the agglomerates of powdered MWCNTs and their dispersions in surfactant solutions. The MWCNT dispersions were sprayed on an aluminum substrate, and SEM micrographs were recorded by the instrument software.

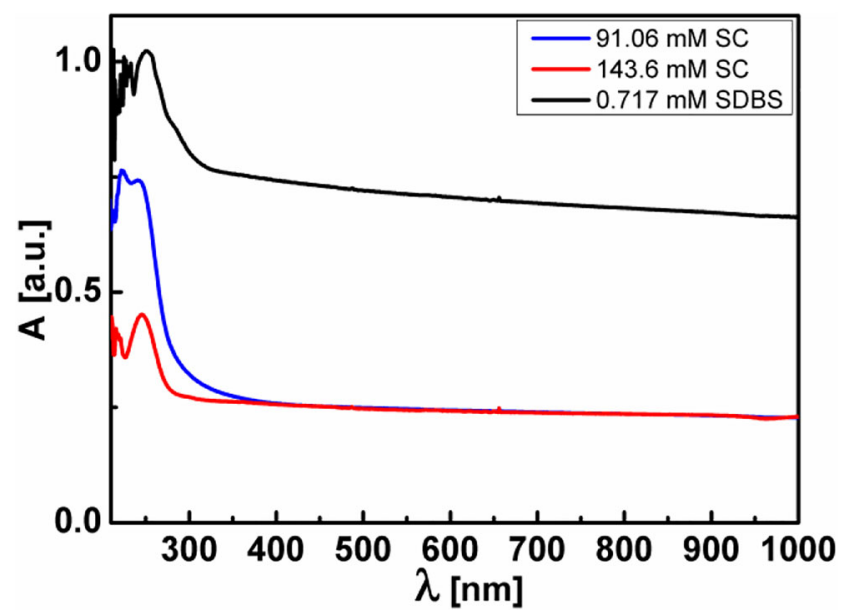

Fig. 4 Absorption spectra of surfactant-MWCNT dispersions at constant MWCNT concentration $(44.3 \mathrm{mg} / \mathrm{L})$ and different surfactant concentrations
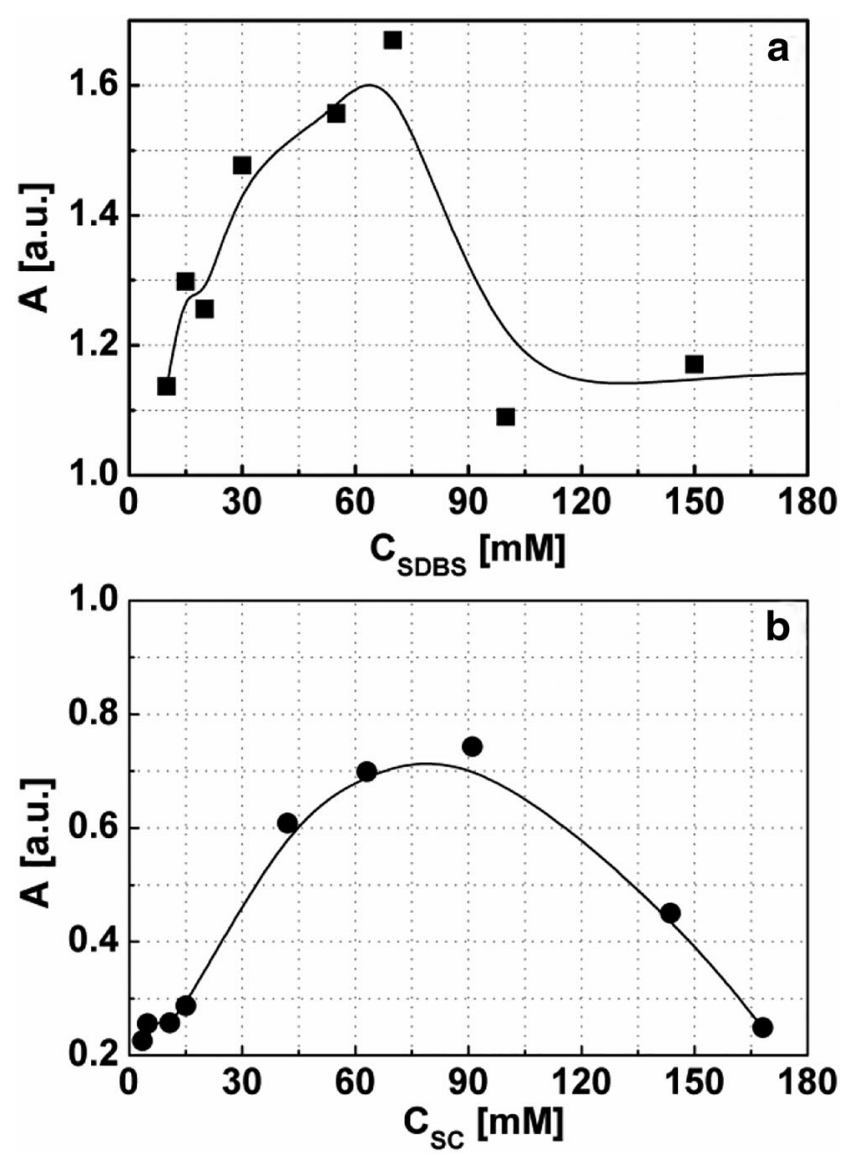

Fig. 5 Variation of absorbance at $273 \mathrm{~nm}$ with the surfactant concentration $\left(\mathrm{C}_{\mathrm{SDBS}}\right.$ for sodium dodecylbenzene sulfonate and $\mathrm{C}_{\mathrm{SC}}$ for sodium cholate) at constant concentration of MWCNT $=44.3 \mathrm{mg} / \mathrm{L}$.

\section{Results and discussion}

\subsection{Microstructure and morphology of carbon nanotubes}

The transmission electron micrographs of pristine multiwalled carbon nanotubes (Fig. 1) demonstrate their cylindrical morphology where graphene sheets are rolled up in a hollow rodlike structure with high aspect ratio $(L / d)$ of about 100 with an average length $(L) \sim 10 \mu \mathrm{m}$ and diameter $(d) \sim 100 \mathrm{~nm}$. The small defected regions along the nanotubes length and their pentagon-like tip (Fig. 1b) confirm the picture of a perfect carbon nanotube that is a cylindrical graphene sheet with seamless structure composed of hexagons with a minimum of defects at the tips [53].

The strong van der Waals attraction forces render carbon nanotubes exist in the form of large agglomerates of size in the range of few hundreds of micrometers (Fig. 2a). Such agglomerates are composed of small (few micrometers) polydisperse aggregates (bundles) of nanotubes (Fig. 2b). 

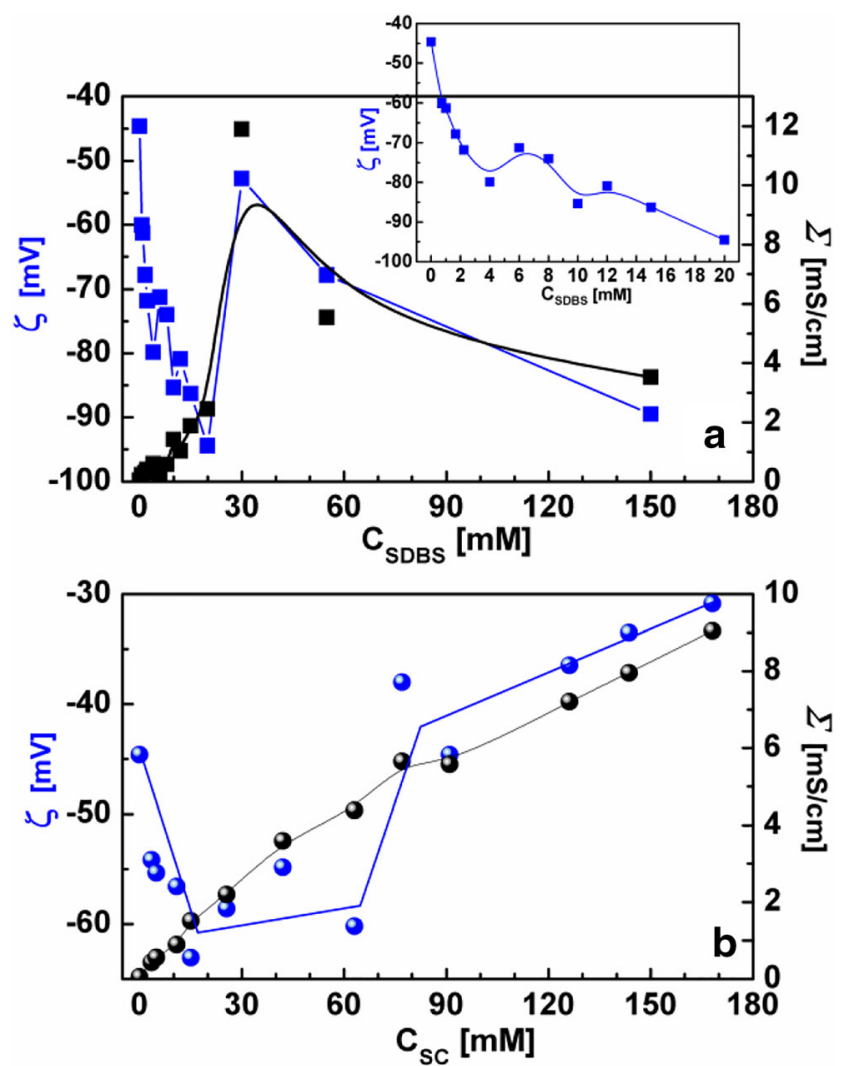

Fig. 6 Variation of zeta potential $(\zeta)$ and ionic conductivity $(\Sigma)$ of dispersions with the surfactant concentration $\left(\mathrm{C}_{\mathrm{SDBS}}\right.$ for sodium dodecylbenzene sulfonate and $\mathrm{C}_{\mathrm{SC}}$ for sodium cholate) at constant concentration of MWCNT $=44.3 \mathrm{mg} / \mathrm{L}$ and $25^{\circ} \mathrm{C}$

\subsection{Nondestructive mild sonication}

In order to obtain good dispersion of MWCNTs in aqueous media, we should overcome the van der Waals attraction forces between the nanotubes. Among various approaches, the utilization of dispersing agents such as low molecular weight amphiphilic surfactants [32] or polymers [30, 54] prove to be efficient and nondestructive method because it is considered as noncovalent functionalization for the surface of nanotubes [55]. Among series of amphiphilic surfactants ranged from ionic to nonionic, anionic sodium dodecylbenzene sulfonate (SDBS) $[25,37-39,41-44,56$, 57] and sodium cholate (SC) [42, 50, 57-59] proved to efficiently disperse MWCNTs in aqueous media.

The sonication is commonly used to facilitate the dispersion of MWCNTs in aqueous media. The input energy should be enough to separate the nanotubes allowing the surfactant molecules to adsorb on the nanotubes' surface. Almost all the research work in this domain relies on using destructive tip sonication that might lead to strong reduction of the length of nanotubes. Then, the dispersibility of MWCNTs may strongly originate from the short nanotubes rather than the surfactant itself. Moreover, the tip sonication usually is conducted in open vials so that solvent evaporation probably occurs and then the concentration of dispersion varies with time. Alternatively, the bath sonication is likely to be a gentle method that aids dispersion of MWCNTs without significant reduction of nanotubes' length, and solvent evaporation is ignored. As can be seen from the SEM micrograph (Fig. 2c), the bathsonicated dispersion of MWCNT in SDBS solution shows individual nanotubes (red arrows) with an average length of $10 \mu \mathrm{m}$ indicating the nondestructive nature of this dispersion method. This sample was sonicated for $2 \mathrm{~h}$; therefore, small bundles of MWCNTs (yellow arrows) still exist in the dispersion. Accordingly, the sonication time $(2 \mathrm{~h})$ is likely to be insufficient to completely disperse the nanotubes.

It is necessarily required to investigate the optimal sonication time that result in better dispersion. Figure 3 demonstrates the effect of sonication time on the absorption spectra of an SDBS-MWCNT dispersion as an example. This dispersion shows an absorption spectrum characterized by an absorption peak at $273 \mathrm{~nm}$. The peak intensity increases with the sonication time indicating a better dispersion quality [56] at sonication time of $8 \mathrm{~h}$ (Fig. 3). This is the first time to systematically investigate the optimal time required to prepare good MWCNT dispersion in comparison to few research studies where bath sonication was used randomly for $2 \mathrm{~h}$ [33] or $24 \mathrm{~h}$ [53]. The 2-h sonication is likely to be insufficient to efficiently disperse the MWCNTs, and the 24-h sonication may induce water evaporation and inaccurate concentration results.

\subsection{Effect of surfactant concentration on MWCNT dispersion: SDBS vs SC}

The quality of MWCNT dispersions in anionic surfactant (SDBS or SC) solutions has been investigated by following the absorption intensity of dispersions. As can be seen from Fig. 4, the MWCNT dispersions show an absorption peak at a wavelength of $273 \mathrm{~nm}$. This peak is characteristic for metallic nanotubes [32] due to the $\pi$-plasmon resonance absorption of $\mathrm{sp}^{2}$-hybridized bonds [35]. Previous studies reported similar absorption peaks over wavelength range from 253 to $300 \mathrm{~nm}$ $[33,34,53,56]$. Such absorption peak signifies individually dispersed nanotubes. Moreover, the absorption spectra lack peaks in the near IR region $(>800 \mathrm{~nm})$ which characterize bundled nanotubes [53]. Figure 4 depicts examples of few surfactant-MWCNT dispersions at fixed amount of MWCNT $(44.3 \mathrm{mg} / \mathrm{L})$ and varied surfactant (SDBS or SC) concentrations.

In an attempt to investigate the effect of surfactant type and concentration on the dispersion quality, the variation of absorbance A (at $273 \mathrm{~nm}$ ) with the surfactant concentration is depicted in Fig. 5 considering the absorption intensity as a measure for the quality of dispersion [53]. At constant CNT concentration $(44.3 \mathrm{mg} / \mathrm{L})$, the absorbance initially increases 


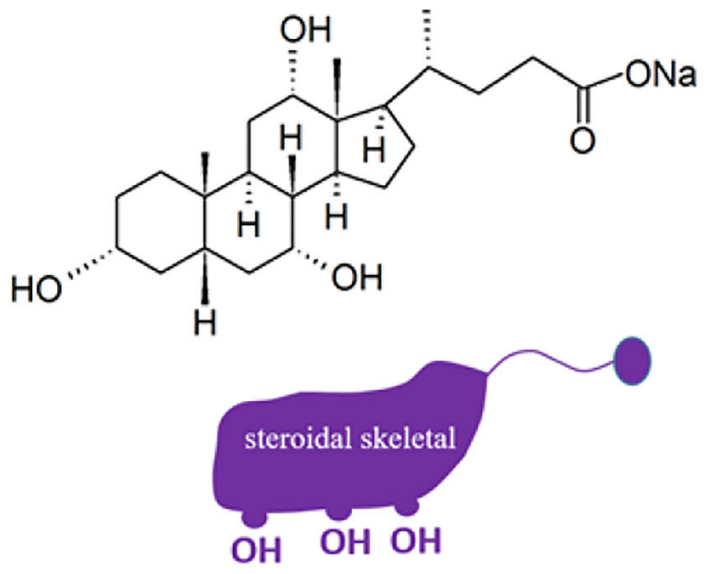

a<smiles>CCCCCCCCCCCCc1ccc(S(=O)(=O)O[Na])cc1</smiles>

\section{Sodium dodecylbenzene sulfonate (SDBS)}

\section{Sodium cholate (SC)}

(I)

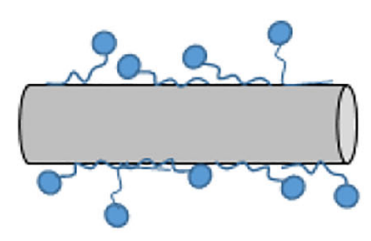

(II)

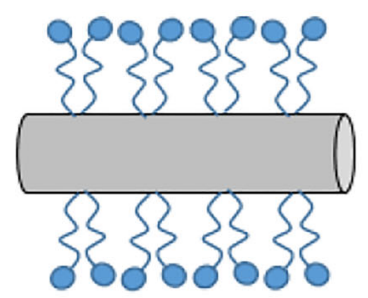

(III)

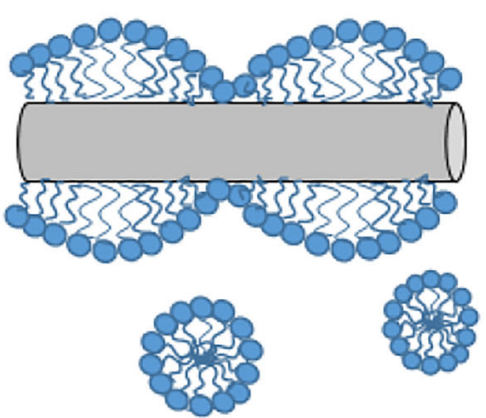

Fig. 7 a Scheme of the chemical formula and diagrammatic structures of sodium cholate (SC) and sodium dodecylbenzene sulfonate (SDBS). b Schematic representations for possible dispersion mechanism for

with the surfactant concentration until reaching a critical concentration beyond which the absorbance decreases. Both anionic surfactants almost exhibit the same trend with different critical concentration. The SDBS-MWCNT system shows a critical concentration at about $70 \mathrm{mM}$ SDBS (Fig. 5a), whereas higher concentration ( $90 \mathrm{mM} \mathrm{SC}$ ) is required to obtain good dispersibility of nanotubes for SC-MWCNT system (Fig. 5b).

Zeta potential $(\zeta)$ is a measure for the surface charge of nanotubes in their aqueous dispersions. It is a valuable physical parameter through which the effect of surfactant on the dispersion mechanism and quality of surfactant-CNT systems can be studied. Figure 6 represents the dependency of zeta potential and ionic conductivity $(\Sigma)$ of MWCNTs with surfactant concentration at constant concentration of MWCNT $(44.3 \mathrm{mg} / \mathrm{L})$. The pristine MWCNT aqueous dispersions (without surfactant) have $\zeta=-45 \mathrm{mV}$ indicating the negative surface charges of nanotubes probably due to ionized
MWCNT induced by surfactants: (I) random adsorption, (II) cylindrical micelles, and (III) hemimicelles on the MWCNT surface (adapted from [60])

carboxylic $(-\mathrm{COOH})$ groups. On addition of anionic surfactant to the MWCNT, the hydrophobic tails of surfactant molecules are expected to wrap (cover) the nanotubes' surface leaving the anionic headgroups exposed to the bulk water. Therefore, the nanotube surface charges are likely to increase, and hence the absolute value of zeta potential $|\zeta|$ increases. This behavior is reasonable below the critical micelle concentration $(\mathrm{cmc})$ of surfactants where the dispersion mechanism is dominated by surfactant wrapping on the surface of the nanotubes by hydrophobic interaction [38]. Above the cmc, different behavior can be recognized depending on the surfactant's concentration and type.

The inset of Fig. 6a presents the dependency of $\zeta$ on the SDBS concentration in the low concentration regime where $|\zeta|$ value dramatically increases from 45 to $80 \mathrm{mV}$ at about $4 \mathrm{mM}$ SDBS beyond which it again slightly decreases to $70 \mathrm{mV}$ then increases to $95 \mathrm{mV}$ at $20 \mathrm{mM}$ SDBS. In the high SDBS 
concentration regime (Fig. 6a), $|\zeta|$ strongly decreases at $30 \mathrm{mM}$ SDBS before increasing again as the SDBS concentration increases. This complex behavior of $\zeta$ with the surfactant concentration is related to different dispersion mechanisms ranged from random adsorption to dispersion stabilized by micelle formation as the surfactant concentration varies. This will be described in Section 3.4. Moreover, the ionic conductivity $(\Sigma)$ surprisingly shows nonmonotonic behavior in tandem with the zeta potential variation with SDBS concentration (Fig. 6a).

The zeta potential of SC-MWCNT dispersions exhibits different trend as the $\mathrm{SC}$ concentration increases, where the $|\zeta|$ strongly increases from 45 to about $65 \mathrm{mV}$ at $15 \mathrm{mM} \mathrm{SC}$ beyond which the $|\zeta|$ continuously decreases with the SC concentration (Fig. 6b). However, the ionic conductivity exhibits continuously increases with SC concentration.

\subsection{Mechanism of dispersion: SDBS vs SC}

It is likely that the two anionic surfactants behave differently because of their different (size and structure) hydrophobic tails (Fig. 7a) and hence different critical micelle concentration (cmc): cmc of SDBS is about $2.23 \mathrm{mM}$ [61], and that of SC is $9-15 \mathrm{mM}$ [62] at room temperature (from $20^{\circ} \mathrm{C}$ to $25^{\circ} \mathrm{C}$ ).

It should be stated that the sign and magnitude of zeta potential respectively reflect the nature and magnitude of surface charges on the surface of nanotubes. Hence, both pristine and surfactant-CNT systems are negatively charged because of $-\mathrm{COOH}$ (for pristine) and the adsorbed anionic surfactant molecules on the nanotube surface. Rational increase of the magnitude of $\zeta$ is expected as the surfactant concentration increases. However, this trend is not monotonic over wide surfactant concentration range as described above. This behavior can be ascribed to different dispersion mechanisms depending on the surfactant molecular structure and its $\mathrm{cmc}$. In addition, it should be noted that the actual cmc of surfactants will be shifted to higher values due to some surfactant molecules adsorbed on the nanotubes. Considering that better dispersion is obtained when $\zeta$ value is maximal $[35,57]$, the mechanism of CNT dispersion can be explained as follows. In the low surfactant concentration regime ( $>4 \mathrm{mM}$ SDBS and $15 \mathrm{mM} \mathrm{SC}$ ), the CNT dispersion is dominated by simple adsorption (Fig. 7b (I)) of surfactant molecules driven by hydrophobic interaction between the surfactant tail and nanotube surface [38]. It is likely that the presence of benzene ring in the tail of SDBS significantly enhances the dispersion by $\pi-\pi$ stacking [32] in comparison to the steroidal moiety of SC so that maximal zeta potential is obtained with SDBS. Increase of the surfactant concentration beyond its cmc results in dispersions stabilized almost by formation of hemimicelles on the nanotube surfaces (Fig. 7b (III)) and hence the zeta potential increases. Further increase of surfactant concentration is likely to induce the formation of cylindrical (rodlike) micelles [31] leading to strong decrease in zeta potential and also ionic conductivity as a result of rendered mobility of ions [62] because of structured aggregates.

\section{Conclusion}

Homogenous dispersions of multi-walled carbon nanotubes (MWCNTs) can be obtained via electrostatic stabilization imposed by anionic surfactants aided by nondestructive bath sonication where the length and graphitic structure of nanotubes are preserved. The maximal stabilization attains via hemimicelles adsorbed on the surface of nanotubes when the surfactant concentration exceeds its apparent critical micelle concentration $(\mathrm{cmc})$. The benzene ring of sodium dodecylbenzene sulfonate (SDBS) tail stabilizes the SDBS-MWCNT dispersion via $\pi-\pi$ stacking interaction resulting in optimal dispersibility at $20 \mathrm{mM}$ SDBS (10 times higher than $\mathrm{cmc}$ ).

Funding information Open Access funding provided by the Qatar National Library. The authors would like to acknowledge the financial support from the Qatar National Research Fund (QNRF) under the grant no. UREP18-172-2-070 and would like to thank the technical staff at the Central Lab Unit and Center for Advanced Materials, Qatar University, for their support in performing SEM, TEM, and Zeta potential measurements.

\section{Compliance with ethical standards}

Conflict of interest The authors declare that they have no conflict of interest.

Open Access This article is licensed under a Creative Commons Attribution 4.0 International License, which permits use, sharing, adaptation, distribution and reproduction in any medium or format, as long as you give appropriate credit to the original author(s) and the source, provide a link to the Creative Commons licence, and indicate if changes were made. The images or other third party material in this article are included in the article's Creative Commons licence, unless indicated otherwise in a credit line to the material. If material is not included in the article's Creative Commons licence and your intended use is not permitted by statutory regulation or exceeds the permitted use, you will need to obtain permission directly from the copyright holder. To view a copy of this licence, visit http://creativecommons.org/licenses/by/4.0/.

\section{References}

1. N.-H. Tai, M.-K. Yeh, J.-H. Liu, Carbon 42, 2774 (2004)

2. N.R.F.J. Kong, C. Zhou, M.G. Chapline, S. Peng, K. Cho, H. Dai, Science 287, 5 (2000)

3. T. Chen, L. Dai, Mater. Today 16, 272 (2013)

4. M. Endo, C. Kim, K. Nishimura, T. Fujino, K. Miyashita, Carbon 38, $183(2000)$

5. M.F.L. De Volder, S.H. Tawfick, R.H. Baughman, A.J. Hart, Science 339, 535 (2013)

6. S. Iijima, T. Ichihashi, Nature 363, 3 (1993)
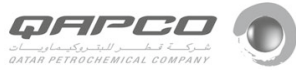

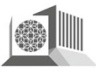

جامعة قطر QATAR UNIVERSITY 
7. J. Chen, L. Yang, H. Yang, J. Dong, Chem. Phys. Lett. 400, 384 (2004)

8. S. Iijima, Nature 354, 3 (1991)

9. M. Zhang, J. Li, Mater. Today 12, 12 (2009)

10. T.W. Ebbesen, Phys. Today 49, 6 (1996)

11. K. Harigaya, Phys. Rev. B 45, 12071 (1992)

12. Y. Ando, X. Zhao, H. Shimoyama, G. Sakai, K. Kaneto, Int. J. Inorg. Mater. 1, 77 (1999)

13. H.P. Boehm, Carbon 32, 11 (1994)

14. L.A. Girifalco, M. Hodak, R.S. Lee, Phys. Rev. B 62, 7 (2000)

15. H. Stahl, J. Appenzeller, R. Martel, P. Avouris, B. Lengeler, Phys. Rev. Lett. 85, 4 (2000)

16. M. Youssry, L. Madec, P. Soudan, M. Cerbelaud, D. Guyomard, B. Lestriez, Phys. Chem. Chem. Phys. 15, 14476 (2013)

17. M. Youssry, M. Cerbelaud, D. Guyomard, B. Lestriez, J. Power Sources 274, 424 (2015)

18. K.L. Lu, R.M. Lago, Y.K. Chen, M.L.H. Green, P.J.F. Harris, S.C. Tsang, Carbon 34, 814 (1996)

19. F. Liu, X. Zhang, J. Cheng, J. Tu, F. Kong, W. Huang, C. Chen, Carbon 41, 2527 (2003)

20. M. Nadler, T. Mahrholz, U. Riedel, C. Schilde, A. Kwade, Carbon 46, 1384 (2008)

21. Y.Y. Huang, E.M. Terentjev, Polymers 4, 275 (2012)

22. T. Saito, K. Matsushige, K. Tanaka, Physica B 323, 280 (2002)

23. Z. Kónya, J. Zhu, K. Niesz, D. Mehn, I. Kiricsi, Carbon 42, 2001 (2004)

24. V. Datsyuk, M. Kalyva, K. Papagelis, J. Parthenios, D. Tasis, A. Siokou, I. Kallitsis, C. Galiotis, Carbon 46, 833 (2008)

25. V.C. Moore, M.S. Strano, E.H. Haroz, R.H. Hauge, R.E. Smalley, J. Schmidt, Y. Talmon, Nano Lett. 3, 1379 (2003)

26. M.O. Lisunova, N.I. Lebovka, O.V. Melezhyk, Y.P. Boiko, J. Colloid Interface Sci. 299, 740 (2006)

27. R. Bandyopadhyaya, E. Nativ-Roth, O. Regev, R. YerushalmiRozen, Nano Lett. 2, 25 (2002)

28. A. Star, Y. Liu, K. Grant, L. Ridvan, J.F. Stoddart, D.W. Steuerman, M.R. Diehl, A. Boukai, J.R. Heath, Macromolecules 36, 553 (2003)

29. F. Cheng, A. Adronov, Chem. Eur. J. 12, 5053 (2006)

30. W. Yi, W. Yi, A. Malkovskiy, Q. Chu, A.P. Sokolov, M.L. Colon, M. Meador, Y. Pang, J. Phys. Chem. B 112, 12263 (2008)

31. B. Vigolo, A. Pénicaud, C. Coulon, C. Sauder, R. Pailler, C. Journet, P. Bernier, P. Poulin, Science 290, 1331 (2000)

32. M.J. O'Connell, S.M. Bachilo, C.B. Huffman, V.C. Moore, M.S. Strano, E.H. Haroz, K.L. Rialon, P.J. Boul, W.H. Noon, C. Kittrell, J. Ma, R.H. Hauge, R.B. Weisman, R.E. Smalley, Science 297, 593 (2002)

33. L. Jiang, L. Gao, J. Sun, J. Colloid Interface Sci. 260, 89 (2003)

34. J. Yu, N. Grossiord, C.E. Koning, J. Loos, Carbon 45, 618 (2007)

35. Z. Sun, V. Nicolosi, D. Rickard, S.D. Bergin, D. Aherne, J.N. Coleman, J. Phys. Chem. C 112, 10692 (2008)

36. F. Tardani, C. La Mesa, Colloids Surf. A Physicochem. Eng. Asp. 443, 123 (2014)
37. M.F. Islam, E. Rojas, D.M. Bergey, A.T. Johnson, A.G. Yodh, Nano Lett. 3, 269 (2003)

38. O. Matarredona, H. Rhoads, Z. Li, J.H. Harwell, L. Balzano, D.E. Resasco, J. Phys. Chem. B 107, 13357 (2003)

39. T. Okazaki, T. Saito, K. Matsuura, S. Ohshima, M. Yumura, S. Iijima, Nano Lett. 5, 2618 (2005)

40. Y. Tan, D.E. Resasco, J. Phys. Chem. B 109, 14454 (2005)

41. S. Utsumi, H. Utsumi, M. Kanamaru, H. Honda, H. Kanoh, H. Tanaka, T. Ohkubo, H. Sakai, M. Abe, K. Kaneko, J. Colloid Interface Sci. 308, 276 (2007)

42. R. Haggenmueller, S.S. Rahatekar, J.A. Fagan, J. Chun, M.L. Becker, R.R. Naik, T. Krauss, L. Carlson, J.F. Kadla, P.C. Trulove, D.F. Fox, H.C. DeLong, Z. Fang, S.O. Kelley, J.W. Gilman, Langmuir 24, 5070 (2008)

43. A.J. Blanch, C.E. Lenehan, J.S. Quinton, J. Phys. Chem. B 114, $9805(2010)$

44. M.D. Clark, S. Subramanian, R. Krishnamoorti, J. Colloid Interface Sci. 354, 144 (2011)

45. M. Qiao, Q. Ran, S. Wu, Appl. Surf. Sci. 433, 975 (2018)

46. P. Angelikopoulos, A. Gromov, A. Leen, O. Nerushev, H. Bock, E.E.B. Campbell, J. Phys. Chem. C 114, 2 (2010)

47. C. A. Kuper, M. Kuzma. US7365100B2 (2008)

48. H. Wang, Curr. Opin. Colloid Interface Sci. 14, 364 (2009)

49. M. Suttipong, N.R. Tummala, B. Kitiyanan, A. Striolo, J. Phys. Chem. C 115, 17286 (2011)

50. W. Wenseleers, I.I. Vlasov, E. Goovaerts, E.D. Obraztsova, A.S. Lobach, A. Bouwen, Adv. Funct. Mater. 14, 1105 (2004)

51. K. Holmberg, B. Jonsson, B. Kronberg, B. Lindman, Surfactants and Polymers in Aqueous Solution (Wiley, West Sussex, 2003)

52. J.F. Offersgaard, H. Öjelund, Appl. Spectrosc. 56, 469 (2002)

53. S. Attal, R. Thiruvengadathan, O. Regev, Anal. Chem. 78, 8098 (2006)

54. M.J. O'Connell, P. Boul, L.M. Ericson, C. Huffman, Y. Wang, E. Haroz, C. Kuper, J. Tour, K.D. Ausman, R.E. Smalley, Chem. Phys. Lett. 342, 265 (2001)

55. D.A. Britz, A.N. Khlobystov, Chem. Soc. Rev. 35, 637 (2006)

56. P. Alafogianni, K. Dassios, S. Farmaki, S.K. Antiohos, T.E. Matikas, N.-M. Barkoula, Colloids Surf. A Physicochem. Eng. Asp. 495, 118 (2016)

57. C. Backes, E. Karabudak, C.D. Schmidt, F. Hauke, A. Hirsch, W. Wohlleben, Chem. Eur. J. 16, 13176 (2010)

58. S. Lin, D. Blankschtein, J. Phys. Chem. B 114, 15616 (2010)

59. M. Gubitosi, J.V. Trillo, A.A. Vargas, N.V. Pavel, D. Gazzoli, S. Sennato, A. Jover, F. Meijide, L. Galantini, J. Phys. Chem. B 118, 1012 (2014)

60. E.J. Wallace, S.P.S. Mark, Nanotechnology 20, 045101 (2009)

61. M. Bakshi, S. Sachar, N. Mahajan, I. Kaur, G. Kaur, N. Singh, P. Sehgal, H. Doe, Colloid Polym. Sci. 280, 990 (2002)

62. A.G. Hsieh, C. Punckt, S. Korkut, I.A. Aksay, J. Phys. Chem. B 117, 7950 (2013) 